\title{
Correction to: Tropical varieties for exponential sums
}

\author{
Alperen A. Ergür ${ }^{1}$. Grigoris Paouris ${ }^{2}$. J. Maurice Rojas ${ }^{2}$ 드 \\ Published online: 28 May 2021 \\ ๑) Springer-Verlag GmbH Germany, part of Springer Nature 2021
}

\section{Correction to: Mathematische Annalen (2020) 377:863-882 https://doi.org/10.1007/s00208-019-01808-5}

A subsidiary result (Theorem 1.1, on the undecidability of determining membership in the amoeba of $1-x-y$ ) from our original article had an erroneous proof. We give a correct proof. The statement of Theorem 1.1 remains correct. We thank Alexander Rashkovskii for pointing out the error in our original proof. We also correct a small typo in our main theorem on univariate exponential sums (Theorem 1.5).

Let $g(z):=\sum_{j=1}^{t} e^{a_{j} \cdot z+\beta_{j}}$ where $z=\left(z_{1}, \ldots, z_{n}\right) \in \mathbb{C}^{n}, a_{j} \in \mathbb{R}^{n}$, the $a_{j}$ are pairwise distinct, $\beta_{j} \in \mathbb{C}$, and $a_{j} \cdot z$ denotes the usual Euclidean inner product in $\mathbb{C}^{n}$. Let $Z(g)$ denote the zero set of $g$ in $\mathbb{C}^{n}, \mathbf{R e}(w)$ the real part of any complex number $w$, and let $\mathbf{R e}(W):=\left\{\left(\boldsymbol{R e}\left(w_{1}\right), \ldots, \mathbf{R e}\left(w_{n}\right)\right) \mid\left(w_{1}, \ldots, w_{n}\right) \in W\right\}$ denote the real part of any subset $W \subseteq \mathbb{C}^{n}$. Our main results from the original article (Theorems 1.5 , 1.9 , and 1.10) revealed a kind of tropical variety that approximates, within an explicit distance bound, the real part of the complex zero set of such a $g$. Such approximations

Alperen A. Ergür was partially supported by the Einstein Foundation, Berlin. J. Maurice Rojas and Grigoris Paouris were partially supported by NSF grant CCF-1900881.

The original article can be found online at https://doi.org/10.1007/s00208-019-01808-5.

$凶 \quad$ J. Maurice Rojas

rojas@math.tamu.edu

Alperen A. Ergür

alperen.ergur@utsa.edu

Grigoris Paouris

grigoris@math.tamu.edu

1 Mathematics Department, University of Texas at San Antonio, One UTSA Circle, San Antonio, TX 78249, USA

2 Department of Mathematics, Texas A\&M University, TAMU 3368, College Station, TX 77843-3368, USA 
are of considerable interest because zero sets of exponential sums appear in many applications. In particular, determining whether a given point $p \in \mathbb{R}^{n}$ lies in $\operatorname{Re}(Z(g))$ is hard in a complexity-theoretic sense: Restricted versions of this problem, even with $n=1$, are already NP-hard in the classical Turing model of computation (see, e.g., [6] or [2, Thm. 4.2]). On the other hand, checking membership in our tropical approximations is doable in polynomial-time for any fixed $n$ : Please see our original article (Thm. 1.9) and [2, Sec. 4].

Theorem 1.1 of our original article stated that determining $p \stackrel{?}{\in} Z(\mathbf{R e}(g))$ is undecidable in the BSS model of computation over $\mathbb{R}$ [4], already in the very special case $g(z)=1-e^{z_{1}}-e^{z_{2}}$. This means that even if we allow unit-time exact field operations and comparisons over $\mathbb{R}$ (something beyond the capability of a classical Turing machine), it is impossible to decide whether an arbitrary $\left(p_{1}, p_{2}\right) \in \mathbb{R}^{2}$ lies in $\boldsymbol{R e}\left(Z\left(1-e^{z_{1}}-e^{z_{2}}\right)\right)$ within time bounded by a real-valued function of $\left(p_{1}, p_{2}\right)$. Determining membership for certain particular values of $\left(p_{1}, p_{2}\right) \in \mathbb{R}^{2}$ can, of course, be quite simple, e.g., $\left(\log \frac{1}{2}, \log \frac{1}{2}\right)$ or $\left(\log \frac{1}{3}, \log \frac{2}{3}\right)$. Similarly, the famous LindemannWeierstrass Theorem tells us that $e^{p_{1}}+e^{p_{2}}$ is transcendental when $p_{1}, p_{2} \in \mathbb{R}$ are distinct and algebraic. However, checking $e^{p_{1}}+e^{p_{2}} \stackrel{?}{\in} \mathbb{Q}\left(p_{1}, p_{2}\right)$ for arbitrary distinct transcendental $p_{1}, p_{2} \in \mathbb{R}$ - using only finitely many rational operations and inequality checks in $\mathbb{Q}\left(p_{1}, p_{2}\right)$ - is already an open question.

For convenience, we quote Theorem 1.1 below:

Theorem 1.1 Determining, for arbitrary input $p_{1}, p_{2} \in \mathbb{R}$, whether $\left(p_{1}, p_{2}\right)$ lies in $\boldsymbol{R e}\left(Z\left(1-e^{z_{1}}-e^{z_{2}}\right)\right)$ is undecidable in the BSS model over $\mathbb{R}$.

The second sentence of the proof from original article (Sec. 3.1) states falsely that the boundary of a countable union of semi-algebraic sets is contained in the union of their boundaries: A simple counter-example, pointed out by Alexander Raskhovskii, is the union of the segments $[1 / n, 1]$ for $n \in \mathbb{N}$, with boundary point 0 not contained in the union of boundaries $\{1,1 / 2,1 / 3, \ldots\}$. We give a corrected proof of Theorem 1.1 below, and include some basic facts (missing from our original article) about semi-algebraic subsets of the plane.

Remark 1.2 We also point out a small typo in Theorem 1.5 of our original article, which concerned an explicit Hausdorff distance estimate between $\operatorname{Re}(Z(g))$ and a certain kind of tropical variety associated to $g$, in the univariate case: The second inequality should have used a soft " $\leq$ " instead of a strict " $<$ ". So the inequality in question should instead read

$$
\frac{(\log 9) s-\log \frac{9}{2}}{\delta(g)} \leq \frac{(\log 9) t-\log \frac{81}{2}}{\delta(g)}
$$

The proof of Theorem 1.5 in Section 2.2 of our original article requires no change. 


\section{Proving Theorem 1.1}

We recall here some basic facts about halting sets, i.e., sets of inputs on which a BSS machine over $\mathbb{R}$ terminates.

Theorem 1.3 [4, Sec. 2.3, Thm. 1, Pg. 52] The halting set of a BSS machine over $\mathbb{R}$ is a countable union of semi-algebraic sets.

The converse of Theorem 1.3 fails in general: For instance, if $S$ is any countably infinite subset of a transcendence basis for $\mathbb{R}$ over $\mathbb{Q}$, then $S$ can not be the halting set of any BSS machine over $\mathbb{R}$. (One can even write such subsets in terms of infinite series, via an explicit basis found by von Neumann [5] around 1928.) This follows immediately from the following consequence of the development in [4, Sec. 2.3]:

Proposition 1.4 Any countable subset of $\mathbb{R}$ that is the halting set for a BSS machine over $\mathbb{R}$ must be a subset of the algebraic closure of a real extension of $\mathbb{Q}$ of finite transcendence degree.

We thank Lenore Blum, Felipe Cucker, and Mike Shub for pointing out Proposition 1.4. We included Proposition 1.4 to clarify the failure of the converse of Theorem 1.3, but we will not need it henceforth.

Let us also recall the following basic facts about semi-algebraic sets, i.e., the solution sets of finite collections of polynomial inequalities and polynomial equalities in $\mathbb{R}^{n}$ : First, semi-algebraic sets are closed under all Boolean operations (intersection, union, and complement). Also, any semi-algebraic set $S$ admits a natural notion of dimension, via the largest $d$ permitting a semi-algebraic embedding of a real $d$-ball into $S$ (see, e.g., [3, Ch. 5, Sec. 5.3, pp. 170-172]). Some additional qualitative facts we'll also need can be summarized as follows:

Theorem 1.5 Suppose $S \subset \mathbb{R}^{2}$ is semi-algebraic, and $\bar{S}$ and $S^{\circ}$ respectively denote the closure and interior of $S$. Then:

1. $\bar{S}, S^{\circ}$, and $\partial S:=\bar{S} \backslash S^{\circ}$ are semi-algebraic.

2. S has only finitely many connected components, each of which is semi-algebraic.

3. If, in addition, $S$ is a connected curve, then $S$ has only finitely many singularities.

4. Let $\rho: \mathbb{R}^{2} \longrightarrow \mathbb{R}$ denote the projection defined by $\rho(x, y)=x$. Then, continuing Assertion (3), there is an $n_{S} \in \mathbb{N}$ such that all fibers of $\rho$ have cardinality at most $n_{S}$.

Proof The first two assertions of (1)are exactly the content of [3, Ch. 3, Prop.3.1,pg.84]. The final assertion of (1) is then immediate since semi-algebraic sets are closed under Boolean operations by definition.

Assertion (2) is immediate from the notion of cylindrical decomposition. The latter is a refined decomposition of a semi-algebraic set into finitely many (semi-algebraic) connected components, and the existence of such a decomposition is a classical fact: See, e.g., [3, Ch. 5, Thm. 5.6, pg. 163]. In particular, the tameness of fibers from Assertion (4) is also an immediate consequence of cylindrical decomposition.

Assertion (3) is a direct consequence of the notion of semi-algebraic cell stratification of $\mathbb{R}^{2}$ adapted to $S$. The latter is a partition $S$ into finitely many semi-algebraic 
smooth manifolds (here, each diffeomorphic to an open interval or a point) called strata, such that the closure of any stratum is a union of strata. That such stratifications exist (and in much greater generality) is also a classical fact: See, e.g., [3, Ch. 5, Thm. 5.38, pg. 177].

\subsection{The corrected proof of Theorem 1.1}

Let $\mathbb{C}^{*}:=\mathbb{C} \backslash\{0\}$ and let $R:=\operatorname{Re}\left(Z\left(1-e^{z_{1}}-e^{z_{2}}\right)\right)$ and $S:=\left\{(\log |x|, \log |y|) \mid 1-x-y=0 ; x, y \in \mathbb{C}^{*}\right\}$.

Via the equality $\log \left|e^{\alpha+\sqrt{-1} \beta}\right|=\alpha$ (valid for any $\alpha, \beta \in \mathbb{R}$ ) we see that $(x, y) \in$ $\boldsymbol{\operatorname { R e }}\left(Z\left(1-e^{z_{1}}-e^{z_{2}}\right)\right) \Longleftrightarrow(x, y)=\left(\log \left|e^{z_{1}}\right|, \log \left|e^{z_{2}}\right|\right)$ for some $\left(z_{1}, z_{2}\right) \in \mathbb{C}^{2}$ with $1-e^{z_{1}}-e^{z_{2}}=0$. Since the exponential function defines a surjection from $\mathbb{C}$ onto $\mathbb{C}^{*}$ we then clearly have $R=S$.

Now note that $J:=\left\{\left(\left|w_{1}\right|,\left|w_{2}\right|\right) \mid 1-w_{1}-w_{2}=0 ; w_{1}, w_{2} \in \mathbb{C}^{*}\right\}$ is exactly the following semi-infinite strip with corners deleted: $I:=\left\{(x, y) \in \mathbb{R}^{2} \mid-1 \leq\right.$ $y-x \leq 1, x+y \geq 1$, and $x y \neq 0\}$. This is because $w_{1}+w_{2}=1 \Longrightarrow\left|w_{1}+w_{2}\right|=1$, $\left|w_{1}\right|=\left|1-w_{2}\right|=\left|w_{2}-1\right|$, and $\left|w_{2}\right|=\left|1-w_{1}\right|=\left|w_{1}-1\right|$. So by the Triangle Inequality we obtain $\left|w_{1}\right|+\left|w_{2}\right| \geq 1,\left|w_{1}\right| \geq|| w_{2}|-1|$, and $\left|w_{2}\right| \geq|| w_{1}|-1|$, and thus (setting $x=\left|w_{1}\right|$ and $y=\left|w_{2}\right|$ ) we obtain $J \subseteq I$. To see that $I \subseteq J$, assume $(x, y) \in I$ and consider $y_{\theta}:=1+x e^{\theta \sqrt{-1}}$ for $\theta \in[0, \pi]$. Clearly $x>0$. So then $\left|y_{\theta}\right|^{2}=(1+(\cos \theta) x)^{2}+(\sin \theta)^{2} x^{2}=1+2(\cos \theta) x+x^{2}$ is a decreasing differentiable function of $\theta$, with $\left|y_{0}\right|=x+1$ and $\left|y_{\pi}\right|=|x-1|$. Since $|x-1| \leq y \leq x+1$ there must then be a $\theta \in[0, \pi]$ with $y=\left|y_{\theta}\right|$. Letting $w_{1}:=-x e^{\theta \sqrt{-1}}$ and $w_{2}:=y_{\theta}$, we then obtain $w_{1}+w_{2}=1,\left|w_{1}\right|=|x|=x$, and $\left|w_{2}\right|=y$. So we have obtained $I \subseteq J$ and thus $I=J$.

Clearly then, $R$ is simply the image of $I$ under the (differentiable) coordinate-wise logarithm map. In particular, we see that the curve $Y$ defined by $y=\log \left(1+e^{x}\right)$, as $x$ ranges over all of $\mathbb{R}$, is a connected component of the boundary $\partial R$.

By Theorem 1.3, if membership in $R$ is decidable, then $R$ must be a countable union $\bigcup_{i \in \mathbb{N}} S_{i}$ of semi-algebraic sets $S_{i}$. Let $W:=Y \cap([0,1] \times \mathbb{R})$, abusing notation slightly by identifying $\mathbb{C}$ with $\mathbb{R}^{2}$. Then $W$ is compact and uncountably infinite, and thus some $S_{i}$ must have $W \cap S_{i}$ infinite. Note in particular that $W \cap S_{i}^{\circ}=\varnothing$ (since $S_{i}^{\circ}$ is in the interior of $R$ ) and thus (by Theorem 1.5) $S_{i} \backslash S_{i}^{\circ}$ must be a finite union of isolated points and smooth connected semi-algebraic curves. In particular, $S_{i}$ must contain a smooth connected semi-algebraic curve $C$ such that $W \cap C$ is infinite. Recalling that $\rho: \mathbb{R}^{2} \longrightarrow \mathbb{R}$ is the projection defined by $\rho(x, y)=x$, we may assume further that $C$ is the graph of a smooth algebraic function $f$ on a non-empty open sub-interval of $(0,1)$, via the Implicit Function Theorem and Assertion (4) of Theorem 1.5. (In particular, this might entail replacing $C$ with a non-empty, connected (and semi-algebraic), open subset of $C$.)

Now observe that $W \cap C$ (resp. $\rho(W \cap C))$ must have at least one accumulation point since $W$ (resp. $[0,1]$ ) is compact, and thus the graphs of the smooth functions $\log \left(1+e^{x}\right)$ and $f$ agree on an infinite sequence of points with a limit point. But this is impossible, since $\log \left(1+e^{x}\right)$ is a transcendental function. In particular, since 
$\log \left(1+e^{x}\right)$ is analytic on the domain $\mathbb{R} \times(-\pi, \pi)$, the function $f$ must have an analytic continuation to an algebraic function with an essential singularity at $\infty[1$, Pg. 127]. Since algebraic functions can only have zeroes or poles of finite fractional order at $\infty$, we obtain a contradiction.

Acknowledgements We thank Alexander Rashkovskii for catching our earlier mistake. We also thank Lenore Blum, Felipe Cucker, and Mike Shub for introducing us to Proposition 1.4. We also point out that while the elementary argument for the equality of the sets $I$ and $J$ (from our corrected proof of Theorem 1.1 here) is most likely folkloric, we first learned of this argument from Mikhael Passare, some time before 2009.

\section{Declarations}

Conflict of interest On behalf of all authors, the corresponding author states that there is no conflict of interest.

\section{References}

1. Ahlfors, L: Complex Analysis, McGraw-Hill Science/Engineering/Mathematics, 3rd edn (1979)

2. Avendaño, M., Kogan, R., Nisse, M., Rojas, J.M.: Metric estimates and membership complexity for archimedean amoebae and tropical hypersurfaces. J. Complex. 46, 45-65 (2018)

3. Basu, S., Pollack, R., Roy, M.-F.: Algorithms in Real Algebraic Geometry, Algorithms and Computation in Mathematics (Book 10), 2nd edn. Springer (2006)

4. Blum, Lenore, Cucker, Felipe, Shub, Mike, Smale, S.: Complexity and Real Computation. Springer, New York (1998)

5. von Neumann, J.L.: Ein system algebraisch unabhängiger Zahlen. Math. Ann. 99, 134-141 (1928)

6. Plaisted, D.A.: New NP-hard and NP-complete polynomial and integer divisibility problems. Theor. Comput. Sci. 31(1-2), 125-138 (1984)

Publisher's Note Springer Nature remains neutral with regard to jurisdictional claims in published maps and institutional affiliations. 